\title{
Qualitative investigation into students' use of divergence and curl in electromagnetism
}

\author{
Laurens Bollen, ${ }^{1, *}$ Paul van Kampen, ${ }^{2, \dagger}$ Charles Baily, ${ }^{3, \ddagger}$ and Mieke De Cock ${ }^{1, \S}$ \\ ${ }^{1}$ KU Leuven, Department of Physics and Astronomy \& LESEC, \\ Celestijnenlaan 200c, 3001 Leuven, Belgium \\ ${ }^{2}$ Centre for the Advancement of STEM Teaching and Learning \& School of Physical Sciences, \\ Dublin City University, Glasnevin, Dublin 9, Ireland \\ ${ }^{3}$ School of Physics and Astronomy, University of St Andrews, St Andrews KY16 9SS, United Kingdom
}

(Received 8 June 2016; published 19 October 2016)

\begin{abstract}
Many students struggle with the use of mathematics in physics courses. Although typically well trained in rote mathematical calculation, they often lack the ability to apply their acquired skills to physical contexts. Such student difficulties are particularly apparent in undergraduate electrodynamics, which relies heavily on the use of vector calculus. To gain insight into student reasoning when solving problems involving divergence and curl, we conducted eight semistructured individual student interviews. During these interviews, students discussed the divergence and curl of electromagnetic fields using graphical representations, mathematical calculations, and the differential form of Maxwell's equations. We observed that while many students attempt to clarify the problem by making a sketch of the electromagnetic field, they struggle to interpret graphical representations of vector fields in terms of divergence and curl. In addition, some students confuse the characteristics of field line diagrams and field vector plots. By interpreting our results within the conceptual blending framework, we show how a lack of conceptual understanding of the vector operators and difficulties with graphical representations can account for an improper understanding of Maxwell's equations in differential form. Consequently, specific learning materials based on a multiple representation approach are required to clarify Maxwell's equations.
\end{abstract}

DOI: 10.1103/PhysRevPhysEducRes.12.020134

\section{INTRODUCTION}

Undergraduate physics students are often expected to already be proficient with requisite mathematical tools. However, education research has shown that many students struggle to apply their acquired mathematical skills in physics contexts [1-11]. Consequently, the investigation of how physics students use mathematics has been a prominent topic in recent literature [12-25]. It is also well established that students have difficulty coordinating information drawn from texts, equations, symbols, graphs, and figures when formulating a solution to a given problem. Nevertheless, it has been argued that the use of multiple representations can aid students in problem solving and with learning concepts [26-34].

Our research project addresses student difficulties with vector calculus before, during, and after an intermediate undergraduate electrodynamics course. In previous exploratory studies, we used a pre- and post-test approach to

\footnotetext{
*Laurens.Bollen@kuleuven.be

†Paul.van.Kampen@dcu.ie

tcrb6@st-andrews.ac.uk

${ }^{\S}$ Mieke.DeCock@kuleuven.be
}

Published by the American Physical Society under the terms of the Creative Commons Attribution 3.0 License. Further distribution of this work must maintain attribution to the author(s) and the published article's title, journal citation, and DOI. gauge student understanding of vector calculus in both mathematics and electromagnetism contexts [1,3]. We demonstrated that most students could adequately carry out rote calculations, but lacked structural understanding of vector operators, struggled with interpreting graphical representations, and often failed to apply Maxwell's equations in differential form correctly.

The qualitative study presented here extends this research by investigating the reasoning processes used by students when solving problems involving divergence and curl of electromagnetic fields. Using a think-aloud protocol, we conducted eight semistructured individual interviews with students who had recently completed an intermediate-level electrodynamics course. The overall aims of this study are threefold: (i) to acquire insight into student reasoning surrounding the use of vector operators in electromagnetism; (ii) to identify specific cues that help students develop an appreciation for the role of divergence and curl in Maxwell's equations; and (iii) to analyze and interpret student interview responses within the cognitive framework of conceptual blending.

In Sec. II, we describe the conceptual blending framework and explain how it can be adapted to make sense of the way students use mathematical knowledge in a physics context. The methodology of the qualitative study is discussed in Sec. III, including descriptions of the data collection, interview protocol, and analysis procedure. Three case studies are presented in Sec IV to demonstrate 
how the analysis procedure is applied in practice, and to give some specific examples of student reasoning employed during the interviews. The results of the study and our observations are discussed in Sec. V, where we also consider to what extent the conceptual blending framework is suitable for interpreting the results. Sec. VI summarizes our conclusions and potential implications for instruction.

\section{THE CONCEPTUAL BLENDING FRAMEWORK}

In the same way that we try to make sense of natural phenomena through models in physics, it is useful to organize findings in education research by using a cognitive model. There are several frameworks that can be used by researchers to gain insight into how students use mathematics in physics. [17-25] These frameworks are often not mutually exclusive and may even strongly overlap. Therefore, there is not a single best or most suitable model to describe how students use vector calculus in electrodynamics. In this paper, we look at the results from student interviews through the lens of the conceptual blending framework.

Conceptual blending, sometimes called mental space integration, was introduced by Fauconnier and Turner to model how people create new meaning in linguistic contexts by selectively combining information garnered from previous experiences [35-39]. A mental space is comprised of conceptual packets or knowledge elements that tend to be activated together, and has an organizing frame that specifies the relationships among the elements within the mental space. It has been suggested that from a neurobiological perspective, mental spaces are built up and interconnected in the working memory by activating structures from the long-term memory, and can be modified as thought and discourse unfold [37]. According to the conceptual blending framework, two or more input mental spaces that share content or structure can be combined into a new, blended space. Since the elements and organizing frames of the input spaces can be blended in a variety of combinations, the projection is a selective one. This implies that the construction of a blended space may strongly depend on cues and context, and that not all elements of each input space necessarily go into the blend. Consequently, the blended space may partially inherit the structure of the input spaces, but also has its own emergent structure. The blending process does not only construct new meaning in the blended space, but may also affect the information in the input spaces.

In the context of physics education research, the conceptual blending theory was first used by Bing and Redish to describe relations between mathematics and physics knowledge during problem solving [22]. Since then, the framework has been applied in other physics contexts to explain how students create new meaning by combining separate mental spaces that share similar content or structure $[14,18,40-42]$. The study conducted by $\mathrm{Hu}$ and

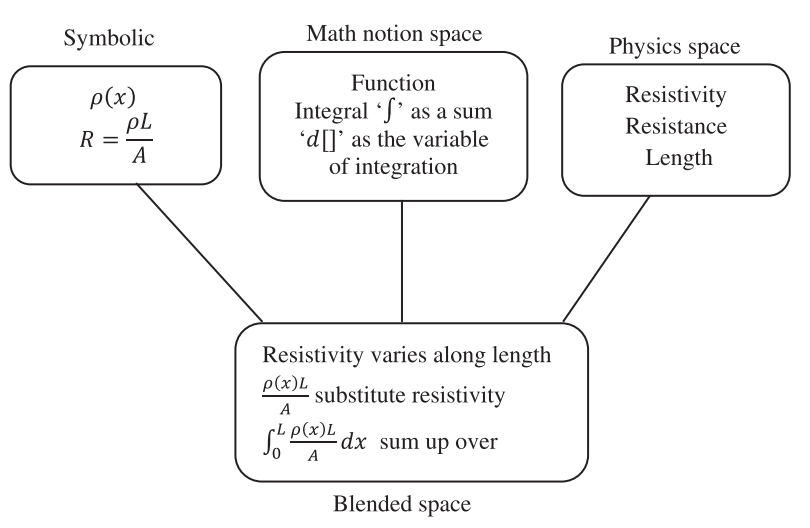

FIG. 1. Example of a blending diagram from the analysis by $\mathrm{Hu}$ and Rebello [18].

Rebello is closely related to our own research project: they adapted the conceptual blending framework to make sense of the ways in which students combine their knowledge from calculus and physics to set up integrals [18].

According to $\mathrm{Hu}$ and Rebello, using mathematics in physics requires the blending of mathematical ideas and symbols with physical situations. They conducted and analyzed group teaching and learning interviews, asking students to calculate the total resistance of a cylindrical wire with a varying resistivity. The conceptual blending framework provided them with more insight into what elements from the input spaces students perceive as relevant and how new meaning is created. An example of such a blend is shown in Fig. 1 [18].

In this paper, the conceptual blending framework is used to gain insight into how students make sense of Maxwell's equations in differential form by combining knowledge from electromagnetism and vector calculus. As will be seen in Sec. V C, there is a direct analogy between the diagrams presented by $\mathrm{Hu}$ and Rebello and the classification of resources that students need to solve questions about divergence and curl in electrodynamics. Therefore, the chosen arrangement of the input mental spaces is copied from the work of $\mathrm{Hu}$ and Rebello, although we share their opinion that there may be other possible classifications. Following their approach, we distinguish between the math notion space, symbolic space, and physics space. The math notion space comprises information and ideas related to mathematical concepts, and so contains all the conceptual ideas a student has about divergence and curl of a vector field, including how these manifest in graphical representations. The symbolic space consists of symbols, notations, and equations that are common to both electromagnetism and vector calculus, including specific formulas for calculating divergence and curl, along with Maxwell's equations in differential form. The physics space is a collection of relevant physical quantities, such as electric and magnetic fields, charge densities, and currents. When students encounter divergence and curl in an electromagnetic 
context, they will selectively use information from these input spaces to create new meaning about Maxwell's equations in differential form.

\section{METHODOLOGY}

\section{A. Participants and data collection}

The individual semistructured interviews were conducted in November and December 2014, with eight students majoring in physics or mathematics who had successfully completed the electrodynamics course during their second Bachelor year. The participants were only aware of the subject of the interview, but not of the exact purpose and were not asked to prepare in any way. Not all of the students took the course in the same academic year or at the same university campus. Consequently, the eight interviewees were taught by four different instructors, all of whom followed the textbook by Griffiths [43]. We did not have access to their final grades, but it was clear from their responses that both high and low achieving students took part. Excerpts from three separate interviews (students A, $\mathrm{B}$, and C) are discussed in detail below. Any quote given without reference to one of the aforementioned pseudonyms is a statement from one of the other five interviewees. The gender of each participant has been maintained.

Each interview lasted approximately one hour, during which the participants were encouraged to "think aloud" as they worked. The interviewer is the first author of the paper, and was at that time not involved in the electrodynamics course. The conversations were audio taped, and video recordings were used to keep track of the drawings and calculations produced by students as they explained their reasoning. Written notes were also drawn up after each interview.

Similar to our approach in the previous study [1], the interviews were used to first identify students' concept images of the divergence and curl operators. A concept image is a unique collection of all the mental pictures, associated properties, and processes that are activated when a person encounters a particular concept [44]. Each participant was then asked to solve physics problems involving the divergence and curl of electromagnetic fields, using a variety of representations. When necessary, hints were provided in order to facilitate discussion.

\section{B. Interview protocol}

Following a preliminary background discussion, each interviewee was asked to give a conceptual description of the divergence and curl operators, then solve several problems that entailed identifying where the divergence and curl of an electromagnetic field might be zero or nonzero. The questions are listed below in the same sequence as they were asked. Clarifications were provided to students when necessary. Follow-up questions allowed us to explore the reasoning process of each participant more fully.

(1) Discuss "the divergence": formulas, descriptions, definitions, etc.

(2) Discuss where the divergence of the electric field is zero and where it is nonzero in the following situations:

(a) the electric field due to a charged infinite insulating cylinder,

(b) the electric field due to a charged spherical shell,

(c) the electric field due to an electric dipole,

(d) the electric field due to a changing magnetic field.

(3) Discuss "the curl": formulas, descriptions, definitions, etc.

(4) Discuss where the curl of the electric field due to an electric dipole is zero and where it is nonzero.

(5) Discuss where the divergence or curl of the magnetic field is zero and where it is nonzero in the following situations:

(a) the magnetic field due to a thin infinite current carrying plate,

(b) the magnetic field due to a charging parallelplate capacitor, if the current in the circuit is assumed to be constant,

(c) the magnetic field due to a solenoid carrying a constant current.

The qualitative nature of the interviews allowed us to further explore the reasoning behind student responses to questions discussed in previous studies. Questions 1 and 3 are extended versions of the first question on our written pretest; the situations in questions 2,4 , and 5 are very similar to those described in the last question on the written post-test [1]. Pepper et al. asked a midterm exam question that was analogous to question 2(b) [4].

Participants initially attempted to answer each question completely, including all subparts [e.g., questions 2(a)-2(d)], without any intervention from the interviewer. After students had summarized their solution, or made clear they were unsure of how to continue, participants were asked to verify or revise their answers using one or more alternative solution strategies involving different representations: performing an algebraic calculation; interpreting a field vector plot; or applying Maxwell's equations in differential form. For example, after the interviewees had given their initial response to question 2(a), the interviewer showed students a vector plot of the electric field due to the charged cylinder [Fig. 2(a)] and then asked them to perform a calculation. Hints were provided if a student was not acquainted with a particular strategy, or got stuck at any point. If a student struggled to interpret a particular field vector plot for example, a simpler field was discussed, such as Fig. 2(b). The interviewer then drew a square box at an arbitrary location in the field, and explained how one can conclude there is a source somewhere inside this box if the net flux is 


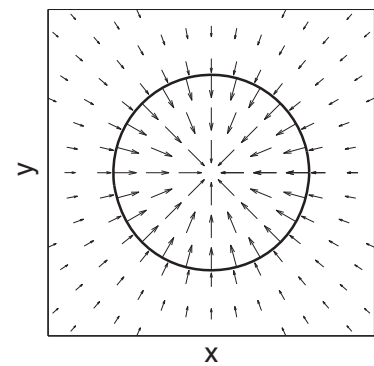

(a)

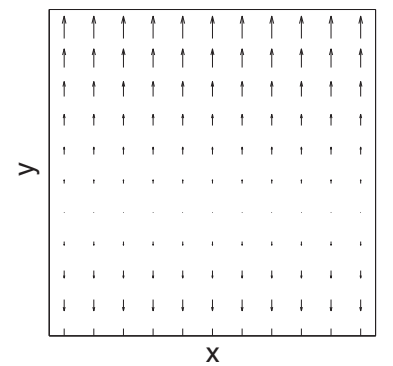

(b)
FIG. 2. Field vector plots that were shown to students during the interview: (a) the electric field corresponding to the charged cylinder of question 2(a); (b) a field used to explain to students how they can decide whether the divergence is zero or not at a certain location.

positive, i.e., more "comes out" than "goes in," without mentioning field lines. Since the size of the box is arbitrary, the correct answer is that the divergence is nonzero everywhere. Afterwards, the interviewee was asked to use the same approach in an electromagnetic context. If a student encountered difficulties while performing a calculation, the interviewer, for example, assisted by pointing out the importance of symmetry in the problem. All necessary formulas, including Maxwell's equations, were provided upon request.

Responses to questions 1 and 3 pertain primarily to a student's math notion space. Questions 2, 4, and 5 intend to gain insight into elements from all mental spaces, including the blended space. Asking students to interpret graphical representations of vector fields can reveal information about their math notion spaces, whereas their performance on calculations can indicate the composition of the symbolic space. In answering questions 2, 4, and 5, students discuss multiple physics concepts included in the physics space, such as charges, currents, and fields. We take a student's ability to interpret and apply Maxwell's equations in differential form as an indicator for how this information has been blended.

During the interviews, we tried not to provide any verbal or nonverbal hints about the correctness of a student's solutions. Feedback on the correctness of their responses was provided only once the interview was over. Afterwards, we reflected back on the interview together with the student; their performance and the aim of the research project were discussed informally. At this stage, most students indicated that they had initially underestimated the difficulty of the questions, but appreciated having gained insight into Maxwell's equations from the experience.

\section{Data analysis}

Shortly after the interviews were conducted, the audio tapes were transcribed. Relevant information from the videos, such as students indicating something on a diagram or references to formulas, was added as commentary in the document. The drawings and calculations made by a student, together with the written notes of the interviewer were used as supplementary material during the analysis.

In our previous work we identified four different skills and competencies students need to acquire concerning vector calculus in electrodynamics: structural understanding $[19,45]$ of the vector operators; interpreting graphical representations of vector fields in terms of divergence and curl; calculating divergence and curl; and conceptual understanding of Maxwell's equations in differential form [1]. Questions 1 and 3 are used to examine the students' structural understanding of the vector operators; questions 2,4 , and 5 are geared towards providing insight into the other skills.

To analyze the interviewees' concept images of divergence and curl, we applied the categorization scheme that emerged from the written pretest data in our previous work [1]. Students'interpretations of the expressions $\boldsymbol{\nabla} \cdot \mathbf{A}$ and $\boldsymbol{\nabla} \times \mathbf{A}$ were used as evidence for conceptual understanding of the vector operators, in addition to their ability to recall correct formulas and the vector or scalar character of divergence and curl. Unlike our previous study, the interviews have allowed us to probe all of these elements in depth, including aspects not initially volunteered by the student. Additionally, the qualitative approach allowed us to investigate to what extent students describe divergence and curl as operators that provide local rather than global information about vector fields.

Questions 2, 4, and 5 presented in Sec. III B can be solved by interpreting a graphical representation, performing a calculation, or applying Maxwell's equations in differential form. We initially only used vector plots as visual representations of the fields, but it became clear during the interviews that many students spontaneously draw field line diagrams instead. We therefore distinguish between the two graphical representations in the analysis below.

We first establish to what extent the interviewees were able to correctly solve problems using the aforementioned approaches. The coding scheme presented in Table I is used to indicate whether a student gave a correct answer straight away, needed some hints, or was not able to arrive at a correct solution. If the student made an error or was unable

TABLE I. The coding scheme used to analyze the correctness of student answers to the questions in Sec. III B.

\begin{tabular}{ll}
\hline \hline Code & \multicolumn{1}{c}{ Description } \\
\hline+ & Correct without any help at all \\
-+ & Incorrect at first but correct after explanation \\
- & Incorrect without further hints, explanation, or revision \\
-- & Incorrect after explanation \\
0 & Mention the strategy without pursuing \\
$\bigcirc$ & Initial idea of the student \\
\hline \hline
\end{tabular}


to progress, a simpler example was given followed by some discussion. The student then attempted to solve the original question a second time using this information. In the coding scheme, the approach initially used by the student has been circled; an encircled " 0 " indicates that a student mentioned a particular strategy but did not follow up on it.

The coding scheme has been applied for each interviewee to all the questions concerning the divergence and curl of electromagnetic fields presented previously in Sec. III B. These data are used to qualitatively discuss the students' ability to switch between approaches, without reference to the correctness of their answers. The next section provides specific examples of conclusions regarding student understanding that can be drawn through the application of this scheme.

\section{CASE STUDIES: PUTTING THE ANALYSIS PROCEDURE INTO PRACTICE}

To illustrate the coding scheme in practice, we present excerpts from interviews with three representative students. These examples provide context for our discussion of how students blend information to make sense of Maxwell's equations in differential form, and indicate specific cues that may help students improve their understanding. In every case, students attempted each of the subquestions individually before any interventions took place.

\section{A. Student A}

When solving the question about the charged cylinder [2(a)], student A initially drew a field line diagram and explained

"I would need to calculate the electric field and the divergence of the field to get the exact result, but since the divergence of the electric field equals the charge density, it will be zero everywhere except for inside the cylinder."

Because student A produced a drawing and mentioned he could perform a calculation, but used Gauss's law to obtain the correct answer, his response is coded as (0) for the field line and calculation approaches, and $\odot$; in the Maxwell's equations category. The coded answers to questions 2(b)-2 (d) are shown in Table II. Because of time constraints, not every question was solved using every approach.

Table II shows that student A was capable of applying multiple solution methods successfully, including a correct use of Gauss's law in differential form. This indicates that selectively blending elements from different input mental spaces presented no conceptual or technical difficulties for this student. Throughout the interview, student A proved to be an examplar for how we would like our students to think about divergence and curl in electrodynamics.
TABLE II. Analysis of responses given by student A regarding the divergence of electric fields [questions 2(a)-2(d)].

\begin{tabular}{lcccc}
\hline \hline & \multicolumn{2}{c}{ Graphical } & & \\
\cline { 2 - 3 } Question & Field vector & Field line & Calculation & Maxwell's Eq. \\
\hline $2(\mathrm{a})$ & + & $(0)$ & $(0)$ & $\oplus$ \\
$2(\mathrm{~b})$ & & & & $\oplus$ \\
$2(\mathrm{c})$ & + & & $\oplus$ \\
2(d) & & $\oplus$ & $(0)$ \\
\hline \hline
\end{tabular}

\section{B. Student B}

Student B is a typical example of someone using a number of approaches in an apparently ad hoc manner. He initially sketched a field line diagram of a charged cylinder [question 2(a)], as shown in Fig. 3, but had difficulty interpreting it correctly.

"The divergence will be positive in this case, I think, since it is a source with field lines coming out of it."

After the interviewer made sure the student meant the divergence is positive everywhere in the field, he added

"I'm not sure if it is positive everywhere, since I lack intuition about this. I should probably calculate it to be sure."

However, when student B answered the other divergence questions 2(b)-2(d), he used a different approach

"Maybe I can apply the theory about the divergence of the electric field here... Like Maxwell's formulas for example... What I'm saying here seems a bit ridiculous."

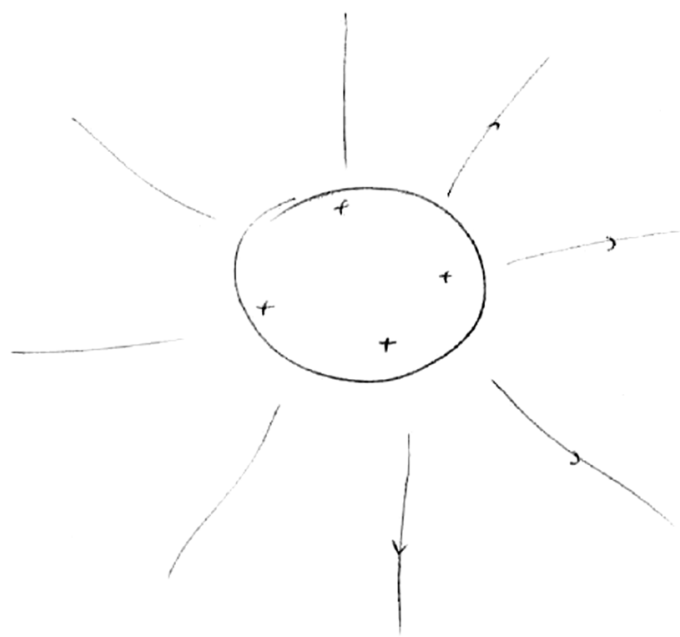

FIG. 3. Student sketch of the electric field of a charged cylinder [question 2(a)]. 
In his first attempt at questions 2(a)-2(d), student B identified all three solution strategies, as seen in Table III. However, unlike student A, he could not get started on a solution to any of the questions without assistance.

The charged cylinder in question 2(a) was discussed in further detail using a field vector plot of the electric field provided by the interviewer [Fig. 2(a)]. However, even after successfully discussing a few simpler examples [e.g., Fig. 2(b)], student B struggled to interpret this particular graphical representation of the electric field due to a charged cylinder. His focus appeared to be on the magnitude of the field rather than on the presence of a field source or sink:

"I might have to compare the length of every arrow to decide about the divergence. That means the divergence will be zero inside the cylinder since all the arrows have the same length, and nonzero outside the cylinder since the length of the arrows increases toward the center."

Student B was then asked to calculate the divergence of this electric field explicitly. At first, he struggled to come up with a correct expression for the field, then with applying the appropriate formula for the divergence. After the interviewer hinted at the cylindrical symmetry of the situation, student B successfully calculated the divergence of the electric field both inside and outside the charged cylinder, and was surprised to find a result that was different from his previous answer. When asked to verify that his result was consistent with Gauss's law, he first turned to the integral forms but struggled to connect this with the divergence of the field. When presented with the differential form of Gauss's law, he said

"Wait, is my calculation correct? [...] So if the charge density is zero, the divergence is zero... But there is a charge in the center, then... I am confused."

An in-depth discussion revealed that student B struggled with the local character of Gauss's law in differential form. At this point, he seemed to think that the charge density being nonzero inside the cylinder implied the field's divergence should be nonzero everywhere, including locations outside the cylinder. When this error was pointed out, he replied that he had once again confused the integral and differential form of Gauss's law. Following this clarification, student $\mathrm{B}$ then realized that the divergence of the electric field is zero outside the cylinder because the charge density is also zero there. In addition, he was now able to use Gauss's law to determine where the divergence of each of the other electric fields 2(b)-2(d) was zero. This development in students B's reasoning is indicated by the -+ signs in the last column of Table III. However, even after this discussion the student still had difficulty understanding why his solution was consistent with the graphical
TABLE III. Analysis of answers given by student B regarding the divergence of electric fields [questions 2(a)-2(d)].

\begin{tabular}{lcccc}
\hline \hline & \multicolumn{2}{c}{ Graphical } & \\
\cline { 2 - 3 } Question & Field vector & Field line & Calculation & Maxwell's Eq. \\
\hline 2(a) & -- & - & & -+ \\
$2(\mathrm{~b})$ & & -- & & -+ \\
2(c) & & & \\
2(d) & & & -+ \\
\hline \hline
\end{tabular}

representations of the field, as indicated by the -- under both graphical representation strategies.

It is valuable to highlight that student $B$ arrived at different results depending on which approach he used. When confronted with his contradictory solutions, he acknowledged that his initial responses must have been based on incorrect ideas. In the conceptual blending framework, this can be described as selecting incorrect information from the input mental spaces to make sense of Gauss's law in differential form. While student B could not identify any specific misunderstandings himself, discussing the differential form of Gauss's law cued an improved understanding of its local nature and, consequently, affected the blending process. When student B subsequently correctly applied Gauss's law to decide where the divergence was zero in three other situations, he used other elements from the input spaces to reorganize his blended space. Nevertheless, despite multiple discussions, his revised blend did not include a correct understanding of how information about the divergence of a field can be deduced from graphical representations. The incorrect and commonly seen student notion of divergence as a measure for how much field lines spread apart, or for how the strength of the field changes with distance, proved to be persistent and was not resolved.

\section{Student C}

In this last example we discuss how student $\mathrm{C}$ solved questions 5(a)-5(c), which involve the curl of a magnetic field. He did not draw a diagram in response to question 5(a), but immediately argued that the magnetic field due to an infinite current carrying plate should be constant, similar to the electric field of an infinite charged plate. Without referencing Maxwell's equations, student $\mathrm{C}$ then reasoned that all derivatives of the field should be zero, and therefore its curl must be zero as well. The charging capacitor and current carrying solenoid questions [5(b)and 5(c)] cued a different approach: in each case he started by drawing a field line diagram and correctly applied Maxwell-Ampère's law in differential form to decide about the curl of the magnetic field. While we have no evidence that unverbalized ideas were not cued at all, it is remarkable that the student used different approaches depending on the 
TABLE IV. Analysis of answers given by student $\mathrm{C}$ regarding the curl of magnetic fields [questions 5(a)-5(c)].

\begin{tabular}{lcccc}
\hline \hline & \multicolumn{2}{c}{ Graphical } & & \\
\cline { 2 - 3 } Question & Field vector & Field line & Calculation & Maxwell's Eq. \\
\hline $5(\mathrm{a})$ & -+ & & - & + \\
$5(\mathrm{~b})$ & & -+ & + & + \\
$5(\mathrm{c})$ & - & - & & $\oplus$ \\
\hline \hline
\end{tabular}

problem presented to him. The corresponding coding can be found in Table IV.

Initially student $\mathrm{C}$ did not give special attention to the actual location of the current carrying plate. When showing him a field vector plot of the situation, he realized the curl is nonzero at the location of the plate using a paddle wheel approach. He then confirmed this idea by taking a closer look at Maxwell-Ampère's law.

While student $\mathrm{C}$ correctly applied Maxwell-Ampère's law in other situations, he indicated that the field line diagrams he had sketched caused him some confusion because they seemed to contradict his conclusions:

"Maxwell's formula is about the current density, but I don't think there is a current density outside of the wires.

However, my drawings seem to suggest that there is some kind of curl."

At this point, student $\mathrm{C}$ could not draw a connection between the symbolic and graphical representations, and was unsure as to which of his ideas were correct. To help him, the interviewer suggested to calculate the curl of the magnetic field due to a charging capacitor [question 5(b)], both between the plates and at an arbitrary location outside of the system. A fluent calculation made him realize that his initial interpretation of Maxwell-Ampère's law was correct, and without further intervention he could identify how an interpretation of a field vector plot would produce the same result:

"This makes sense, if the changing electric field vanishes, the curl of the magnetic field should vanish as well. However, the magnetic field itself is of course nonzero. [...] The drawing confused me at first, but now I can see that a paddle wheel would not rotate here."

He then explained that a paddle wheel would remain stationary in a purely azimuthal $1 / s$ field because the field would "push" on either side with equal strength. While his argumentation was not rigorous, student $\mathrm{C}$ had gained insight into the determination of where the curl is nonzero in a graphical representation of a vector field by improving his understanding of Maxwell-Ampère's law. In the context of the conceptual blending framework, this serves as an example of how a student can acquire a deeper understanding of the elements in the input space by refining the blending process.

Reasoning through these questions with some in-depth guidance significantly enhanced this student's ability to switch between the differential form of Maxwell-Ampère's law, graphical representations, and calculations, and clarified the physical meaning of vector operators in electrodynamics. It also shows how making the student aware of contradicting responses can cue him to reconsider his reasoning and thereby lead to an improved understanding.

\section{RESULTS AND DISCUSSION}

As mentioned in Sec. III B, all eight participants answered three questions, comprised of multiple parts, about divergence and curl of electric and magnetic fields. This resulted in a total of 32 tables similar to the examples discussed above. In this section, we discuss our observations and recurring student difficulties that became apparent through our analysis of the interview data. The first subsection contains information about the concept image of the students (questions 1 and 3). The outcomes of the analysis of the other questions are discussed in the second subsection. In the last subsection, we explain to what extent the conceptual blending framework is suitable to interpret these results.

\section{A. Concept image of the divergence and curl}

When the interviewees were asked to discuss divergence or curl, they typically first tried to write down a formula. Almost every student was able to reproduce a correct mathematical expression for both vector operators in Cartesian coordinates, and concluded from these that both operators are defined locally. However, the argumentation to support this conclusion was typically rather weak or incorrect, for example,

"The components in the formula will not be the same everywhere in the field, so the divergence will change as well... Does that make sense?"

"The divergence depends on the location, since it is bigger where field lines are closer to each other."

This indicates that while most students realize divergence and curl are local operators, many of them may not fully understand what this implies.

The divergence is formally defined as the limit of the net flux across the closed boundary of a volume divided by that volume, as the volume shrinks to zero. The magnitude of the curl is defined as the limit of the circulation along the boundary of an area divided by that area, as the area shrinks to zero. However, in textbooks divergence is often introduced by calculating the dot product of the Nabla operator with a vector field, and the curl is presented as the cross product of the Nabla operator with a vector field. These 
operations result in the mathematical expressions for divergence and curl in Cartesian coordinates. Consequently, students generally treat these expressions as the definitions for the vector operators, and make no notice of the formal definition of divergence and curl. Nevertheless, the formal definitions contain a lot of conceptual information; for example, how the divergence at a certain location in a vector field can be found by determining the flux through a shrinking box. In addition, the partial derivatives in the expression in Cartesian coordinates may support the idea that divergence is a measure for how the magnitude of the field changes with distance. Therefore, we argue it is valuable to discuss the distinction between the formal definition and the mathematical expression explicitly during instruction.

For the remainder of the interview students would often talk about the divergence and the curl. This specific use of wording, that can also be found in many textbooks, can unintentionally contribute to the incorrect idea that these vector operators have a single value that characterizes all of the field. Therefore, it is an example of how one's choice of language can impact student thinking, and so it pays for instructors to be conscious of this and to explicitly attend to this particular misunderstanding. It may also help to omit "the" where possible, as we tried to do in this manuscript. We think it cannot be stressed enough that both divergence and curl generally have different values at different locations.

Most of the interviewees struggled when asked to describe divergence in words. One participant confused the divergence with the gradient, and therefore thought it should be a vector quantity. While the others acknowledged that the divergence is a scalar quantity, they found it difficult to give an accurate description of the actual concept. Some of the explanations contained ideas that were mostly correct, such as the following:

"I remember from the course that this is a measure for what is added or removed from the field at a certain point."

"The divergence is a scalar that measures how much the field changes at a certain point, like derivatives usually do. So in every point I will look how big the change is in the $x, y$ and $z$ direction."

Other students gave completely incorrect descriptions, for example,

"This measures how strongly field lines spread apart. When you have a charge that generates an electric field, there will be a strong divergence since the field lines spread apart as you go further from the charge. In a homogeneous field you will have no divergence. So the divergence is larger where field lines are closer to each other."
While the formula for the curl is rather involved compared to the divergence, most students could reproduce it with the help of a determinant. They also stated that the curl is a vector quantity. All the interviewees gave similar descriptions of the curl, e.g.,

"The curl is a measure for how much the field rotates around a certain point."

While this is not necessarily incorrect, we observed that students often misunderstood the concept when applied in an electromagnetic context. For example, one of the participants made an indication on Fig. 2(a) and explained

"Here the curl is zero, because the vectors are in a straight line. So when you follow the vectors they will never make a turn. But if you look at an electric dipole, the vectors will make a turn, and then there might be a curl... however I'm not sure if that is correct."

During the interviews, only a few students mentioned Stokes'theorem or the divergence theorem. Since investigating students'understanding of these theorems was beyond the research goals, we did not ask them to elaborate about their statements. However, both are important theorems that are closely linked to the formal definition of the vector operators, and it is therefore important to discuss it during instruction. In addition, it may help students to understand how to treat divergence and curl at singularities, for example, at the location of point charges. While an extended discussion on this subject is valuable, it is beyond the scope of this paper.

The data show that many participants relied on vague descriptions of divergence and curl that may or may not contain correct information. These results are in agreement with both the findings from the written pre- and post-tests $[1,3]$ and other studies that report on students' understanding of vector operators $[2,4,46,47]$. This incomplete conceptual understanding of divergence and curl seems to be persistent, since all the interviewees had successfully finished the second year course on electrodynamics. In addition, the remainder of this section will show how misinterpreting vector operators may result in a lack of insight into Maxwell's equations in differential form.

\section{B. Divergence and curl of electromagnetic fields}

In this section we discuss the main difficulties our interviewees encountered when solving problems involving divergence and curl of electromagnetic fields, using one or more of the approaches discussed above. In addition, we explain how certain cues can help students improve their reasoning and understanding. We hope this information will be valuable when designing specific learning materials on vector calculus in electrodynamics. 
Before formulating an answer to questions 2, 4, and 5, most of the interviewees sketched a field line diagram. Every participant used this approach at least once during the interview, but only student A did so successfully. The other seven students often made inaccurate drawings and found it difficult to explain their ideas. Typically they would pay little or no attention to the local character of divergence and curl, like student B in Sec. IV B. Another participant initially sketched a field line diagram and had similar difficulties when trying to interpret it:

"You can see that there certainly is a divergence, both inside and outside the cylinder. The further you move to the center of the cylinder, the higher the density of the field lines becomes. Therefore, the divergence must be nonzero."

It appears that, just like student B, this student confused the derivative of a function with its value. Instead of discussing the change in number of field lines, he only commented on the strength of the field. Many interviewees made similar errors and ended up describing divergence as a measure for spreading field lines and curl as a measure for bending field lines. The following quotes illustrate how these ideas resulted in incorrect statements about the divergence and curl of an electric dipole field [questions 2(c) and 4]. In these cases, students commented on a sketch they made, similar to the one in Fig. 4(a),

"Oh well... I think it should be something like this. You can see that when you are closer to the poles, the field lines are denser. And here they are further from each other... Or my drawing is inaccurate... So I think the divergence will not be equal to zero."

"The curl will be zero where the charges are, but I'm not sure about the rest of the field. I would probably say that where the field lines bend, the curl would be nonzero."

In general, students struggled with similar issues when trying to make sense of field vector plots, e.g.,

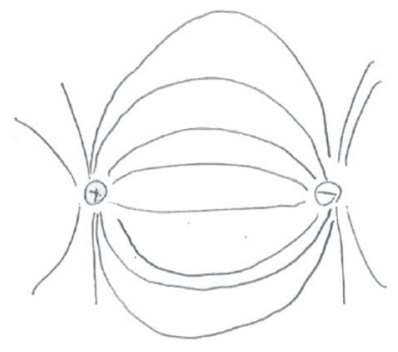

(a) Student sketch of the electric field of a dipole [question 2(c) and 4].

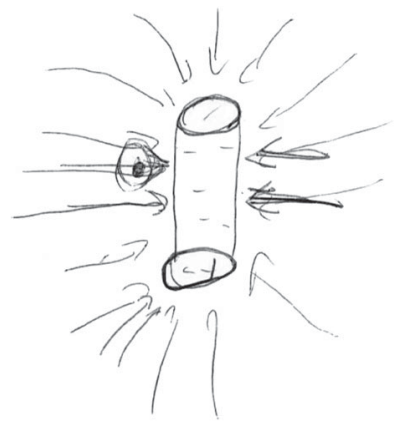

(b) Inaccurate student sketch of the electric field of a charged cylinder [question 2(a)].
FIG. 4. Examples of student drawings of electric fields.
"When the vector arrows all point in the same direction, there is not much of a curl I would say. However, if the direction of the field changes, the curl must be quite strong."

The divergence and curl as measures for the spreading and curvature of a field, respectively, were the most prevalent sources of mistakes when interpreting graphical representations.

It was rather surprising that the interviewees sketched field line diagrams so often, since most instructional materials use field vector plots when graphical examples are provided. However, some students drew arrows that were neither field lines nor field vectors and made incorrect conclusions based on these representations; one such sketch is shown in Fig. 4(b). This may indicate that students are not aware of the differences between field lines and field vectors and do not fully appreciate the strengths and weaknesses of both representations, as suggested in earlier studies $[42,48,49]$. However, during the interviews it was difficult to deduce whether students were confused by the difference between field vector plots and field line diagrams or if they simply had problems to decide about divergence and curl in both representations. Therefore, we plan to investigate to what extent students can switch from field line diagrams to field vector plots and vice versa, and how they determine where the divergence and curl are nonzero in both representations.

Since making a sketch of the situation is an important heuristic in physics problem solving, it is not surprising that students strongly rely on diagrams and drawings. Nevertheless, it is remarkable that the interviewees made drawings despite their difficulties with interpreting graphical representations. Consequently, we want to encourage the use of graphical representations when teaching about vector calculus in electrodynamics, in agreement with the conclusions from other studies $[1,2,4,46,50]$.

As expected, the participants were adequately skilled in doing calculations with the vector operators. However, all interviewees except student $\mathrm{A}$ had difficulties setting up an equation for the field when attempting to calculate divergence or curl. These problems were mostly related to the correct use of the symmetry of the situation. Some students confused spherical and cylindrical symmetry, causing uncertainty about the distance dependence of the discussed electromagnetic fields. In addition, they were unsure which "formula" for div and curl should be used. Nevertheless, after some help, all students were able to calculate the divergence and curl of the electromagnetic fields given.

In general, the interviewees did not remember Maxwell's equations in differential form by heart, so all four laws were eventually provided. Many of the interviewees struggled when asked to interpret these equations. Mistakes were often due to an incorrect interpretation of the locality of the laws, despite all students saying that divergence and curl 
are defined locally. Some of these incorrect ideas are possibly caused by confusing the integral and differential forms of Maxwell's equations. Such issues were already pointed out in Sec. IV B, when discussing how student B made sense of Gauss's law. Another illustration of such a problem was observed when student $C$ explained that he prefers the integral form of Maxwell's equations:

"Personally, I think it is easier to look at an area and see that it changes. [...] The differential form is local and then it is possible that it cancels out at some other points and so on, or at least that is my intuition about it. If you consider the complete region at once, you don't risk forgetting something."

Clearly, student $\mathrm{C}$ also struggled with an incorrect idea related to the local character of Maxwell's equations in differential form. He appeared to assume that while the divergence can have different values in different spatial locations, there should still be some influence from the field in other locations as well. As discussed in Sec. IV C, he had similar issues when tackling questions about the curl of a magnetic field. Such problems with Maxwell-Ampère's law were rather prevalent, as another participant also incorrectly concluded that the curl of a magnetic field should be nonzero everywhere if there is a current density somewhere:

"The curl will be nonzero everywhere here, since the current density $J$ is constant and not zero here (points at the location of the current carrying plate), and there is no changing electric field. So the curl of the magnetic field must be nonzero."

These are just a few examples of many interview excerpts that show how students improperly use the differential form of Maxwell's equations by making an error related to the locality of divergence or curl. Often students realized that the right-hand side of the equations (e.g., $\boldsymbol{\nabla} \times \mathbf{B}=$ $\left.\mu_{0} J+\mu_{0} \epsilon_{0} \partial \mathbf{E} / \partial t\right)$ is local, but they do not treat the lefthand side as local. Therefore, they often conclude that the divergence or curl is nonzero everywhere if there is a nonzero charge density or current density somewhere. Even when students explicitly stated that the magnitude of divergence and curl depends on the location, they still had some problems concerning the local character of Maxwell's equations in differential form.

During the interviews, participants often answered questions differently depending on the approach they used. For example, student B was able to calculate the divergence of the electric field due to a charged insulating cylinder and understood how this was in agreement with Gauss's law, but he did not see the link with graphical representations of the vector field. Similarly, another participant could find some correct answers after a couple of hints about
Maxwell-Ampère's law, but had difficulties explaining these solutions when a field vector plot was presented to him. One of the most disturbing observations was that students not only have difficulties interpreting graphical representations, but sometimes prefer their invalid reasoning above an interpretation of Maxwell's equations or their calculations:

"If you look at the figure, it seems evident that there is a divergence, so I rather think that there is something wrong with the use of the formula than with my interpretation of the sketched figure."

This is remarkable, since most of the participants seemed to be more confident performing a calculation. However, after recalculating and revising their reasoning, most of the interviewees realized their initial interpretation of a field line diagram or field vector plot was incorrect. In about $50 \%$ of the cases, they were able to correct their initial answer and could successfully explain how the graphical representations made sense given their responses based on the other approaches, like student C in Sec. IV C. This indicates that working with multiple representations can cue an improved understanding of Maxwell's equations in differential form.

Students often benefited from situations where the interviewer showed them how different responses contradicted, certainly when they were able to revise their reasoning. While certainly not all the interviewees managed to eventually find correct answers, all of them indicated gaining a lot of insight into Maxwell's equations after the interview. Since the most persistent difficulties are linked to interpreting graphical representations, these should be included in instruction on divergence and curl in electrodynamics. It is also of great importance to teach students how to switch from one representation to another. In our opinion this is key in understanding Maxwell's equations in differential form.

\section{Interpretation of results using the conceptual blending framework}

Using the results in the previous sections and the case studies in Sec. IV, we now model some of the identified problems with the help of the cognitive framework of conceptual blending. As explained in Sec. II, this framework describes how one selectively blends elements from input mental spaces to create new meaning. Interpreting our results within the conceptual blending framework helps us to gain insight into the causes of student difficulties and can therefore be valuable when developing specific learning materials that aim to clarify Maxwell's equations in differential form.

In Sec. IVA we pointed out that student A showed a good understanding of the vector operators and performed a flawless calculation of the divergence. In addition, he 
demonstrated a high degree of acquaintance with physical concepts and quantities. Consequently, the information in his math notion space, symbolic space, and physics space was both complete and correct. The blending diagram in Fig. 5(a) shows how he used certain elements of these input spaces to blend a new space containing a local version of Gauss's law: the divergence of the electric field is nonzero at a certain location if and only if the charge density is nonzero at this location. To emphasize that a student might gain reciprocal insight into the input spaces, the arrows in the blending diagram are bidirectional.

Most interviewees showed a very satisfying familiarity with the physical concepts throughout the interview and performed calculations with the vector operators fairly well. Therefore, the information in the symbolic and physics space was very similar for all participants. However, as discussed before, some students think of divergence as a measure of spreading field lines, which is generally incorrect. Such ideas also showed up during the interview with student B, an excerpt of which was discussed in Sec. IV B. In a blending diagram, this is presented as selecting incorrect information from the math

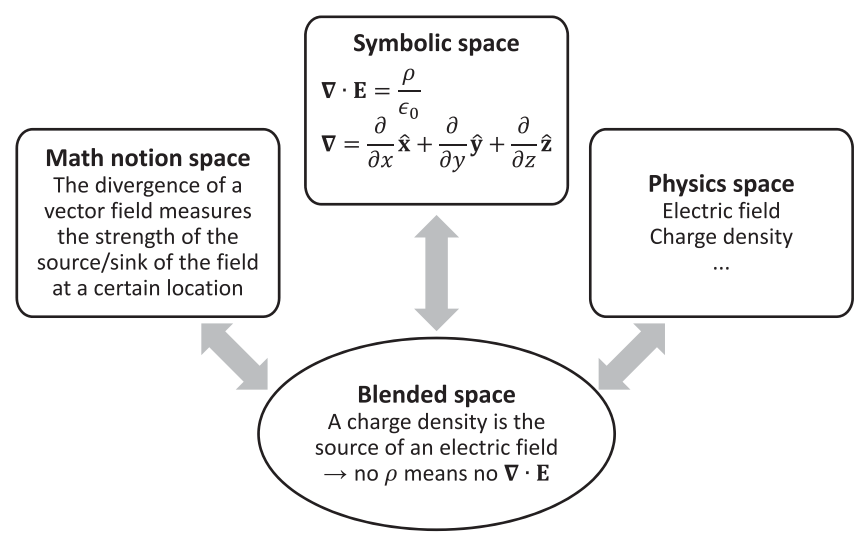

(a) Blend of student A, who showed a good understanding of Gauss's law.

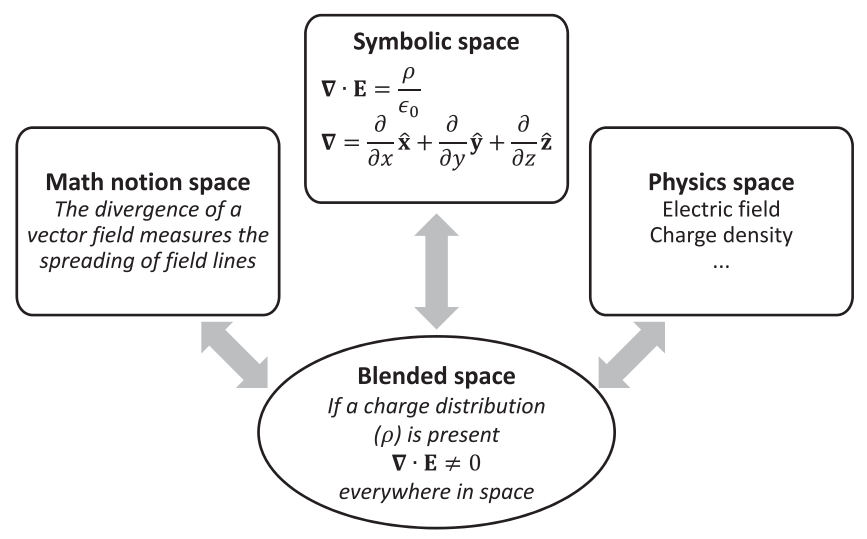

(b) Initial blend of student B, who misunderstood Gauss's law.

FIG. 5. Examples of blends concerning the understanding of the divergence of an electric field. notion space and mapping it into the structure of the symbolic space. The blending diagram that represents how student B initially made sense of Gauss's law in differential form is shown in Fig. 5(a). Since student B's math notion space contained elements different from that of student A, the blended space in Fig. 5(b) differs from the one in Fig. 5(a). More precisely, student B interpreted Gauss's law as follows: if there is a charge distribution somewhere in space, the divergence should be nonzero everywhere since the field "spreads out."

The diagram in in Fig. 5(b) helps us to understand how students may misinterpret Gauss's law in differential form when lacking a good understanding of the concept divergence, including a notion of how divergence is represented in field line diagrams and field vector plots. Analogous mistakes, and thus blending diagrams, appear in the case of Maxwell-Ampère's law. When magnetic field lines circle around a current carrying wire for example, students often think the curl should be nonzero everywhere since the field is generated by a current density. Such an example was already discussed in Sec. IV C. However, after a conversation with the interviewer, student $\mathrm{C}$ revised the information in the blended space and improved his understanding of both curl and Maxwell-Ampère's law. This indicates that students' blending processes can partly be guided, which is in line with the idea that blending depends on certain cues and contexts. Therefore, instruction on vector calculus should include the interpretation of the vector operators in graphical representations of vector fields. In addition, it shows how students can benefit from an increased focus on the relation between field line diagrams, field vector plots, calculations, and the differential form of Maxwell's equations.

The distinction between single-scope blends and doublescope blends is often made in the literature [18,22,36,42]. Single-scope blends import elements from one or more input spaces into the organizing frame of another, for example, when a student maps physical quantities to an existing mathematical equation. On the other hand, doublescope blending involves an integration of organizing frames of input spaces. In the latter case a student would not only use mathematical expressions to map the physical quantities, but would also describe the physical context using a mathematical relation. During the interviews, most of the students, if not all, seemed to use single-scope blends. A clear example of such one-way mapping was shown in student B's blending diagram: he projected the information from the math notion space into the structure of the symbolic space. As Bing and Redish stated, singlescope blends may be typical for mathematically dominated topics, and are not more or less appropriate than doublescoped blends [22]. Nevertheless, a double-scope blend is often related to a deeper understanding of the link between mathematics and physics.

As discussed in the previous paragraphs, the conceptual blending framework is helpful to describe how students use 
divergence and curl in electrodynamics. We explained how the discrepancy in the math notion space of student B caused him to initially misinterpret Gauss's law in differential form. After a second try, however, student B did come up with a satisfying interpretation of Gauss's law in differential form. Nevertheless, as explained in Sec. IV B, he could not link it to the graphical representations of the electric fields. This indicates that the presence of correct elements in the blended space does not necessarily imply a deep understanding of these concepts, since not all the relevant information in the input spaces may have been selected during the blending process. On the other hand, complete and correct information in the input spaces does not automatically result in the intended new meaning after blending. There were multiple participants who gave reasonably accurate descriptions of divergence and curl, but used a very contradictory approach when applying the vector operators in a physics context. This shows that student difficulties may often not be due to a lack of prior knowledge, but may stem from improperly blending the knowledge of mathematics with the symbolic expressions and physics context. Even if all pieces are present, students still need time and resources to finish the jigsaw. In other words, the dynamics of the blending process itself should not be underestimated, as they are not entirely understood yet. In addition, the importance of the learning context in which blending occurs remains uncertain. While this is not denied in literature about the conceptual blending framework $[18,22,35,36,38]$, it might be undervalued when applied to physics problem-solving contexts.

Another element that can partly account for students misinterpreting the locality of the differential form of Maxwell's equations has to do with confusing the integral and differential forms of the laws. In the previous sections, we already discussed in detail how students struggle to make a clear distinction between the two forms. While some participants' reasoning was influenced by the difference between the integral and differential form, it is unclear in which input space this information should be included when interpreting results in the conceptual blending framework. Possibly, these misunderstandings stem from incorrect connections between resources from various mental spaces, but further research is needed to interpret this in the conceptual blending framework.

In conclusion, we believe it is valuable to use the cognitive framework of conceptual blending to describe students' reasoning about divergence and curl of electromagnetic fields, but are aware that certain limitations of the framework may cause student difficulties to be oversimplified or misinterpreted.

\section{CONCLUSIONS AND IMPLICATIONS}

We have presented and discussed the results obtained by conducting semi-structured student interviews about the use of vector operators in electrodynamics. We have shown that most of the interviewees could write down correct formulas for divergence and curl and realized that both operators are defined locally. However, they lacked conceptual understanding, did not distinguish between the mathematical expressions and the formal definitions of the vector operators and encountered difficulties when these concepts had to be applied in an electrodynamic context.

During the interviews, students were asked to discuss the divergence and curl of several electromagnetic fields. Most students initially sketched diagrams of vector fields rather than performing a calculation or applying Maxwell's equations in differential form. However, students'sketches were often incomplete or, drawn without great care, and contained elements from both field line and field vector diagrams, which made it hard for them to decide where divergence and curl are nonzero. In addition, many students struggled to interpret graphical representations because they described divergence as a quantity related to the spreading of field lines, and curl as a measure of how much field lines bend. Whether these problems are caused by misunderstanding the characteristics of field vector plots and field line diagrams or rather the interpretation of divergence and curl in graphical representations is a subject for further research.

While the interpretation of graphical representation was a relatively difficult task for the interviewees, they were generally well trained in performing calculations with the vector operators. Nevertheless, some participants struggled to use the symmetry of the physics context in the questions correctly. When the interviewees applied the differential form of Maxwell's equations to make decisions about divergence and curl, they often made mistakes related to the locality of the laws. This shows that students encounter difficulties with the use of divergence and curl in electrodynamics, regardless of which approach is used. Therefore, it is valuable to discuss all three approaches when teaching about the use of vector calculus in an electromagnetic context.

Frequently, students would obtain different answers depending on which approach they used and had difficulties deciding which solution was correct. Even after a correct calculation or an appropriate use of Maxwell's equations, for example, the interviewees were not easily convinced that their contradicting solutions obtained by misinterpreting graphical representations were incorrect. In addition, they would give more credence to their intuitive ideas than to results based on mathematical principles. Confronting students with these contradictory responses often cued an improved reasoning and understanding of Maxwell's equations in differential form. Therefore, more focus should be put on teaching students how to switch between representations.

Using the conceptual blending framework, we showed how lack of conceptual understanding of the vector operators and misinterpreting graphical representations 
can cause students to misunderstand Maxwell's equations. However, we also pointed out that the blending process itself and additional factors that are difficult to include in the input spaces can have an important influence in the way students think about Maxwell's equations in differential form. This shows that one should be careful not to oversimplify the cognitive processes involved in creating new meaning when applying the conceptual blending framework. Nevertheless, describing students' use of divergence and curl in an electromagnetic context with the conceptual blending framework may help instructors to understand student difficulties in this topic.

Based on these findings, we are designing, implementing, and evaluating learning materials that clarify the use of divergence and curl in electromagnetism. While some available works contain valuable ideas and are excellent choices to use during instruction on vector calculus in electrodynamics [51-53], we opt for a guided-inquiry approach that comprises of worksheets which explicitly tackle the difficulties that were identified in our research project. More specifically, we focus on the conceptual meaning of divergence and curl and use a multiple representation approach to explain Maxwell's equations in differential form. Graphical representations are a vital ingredient in these tutorials, since students heavily rely on vector field visualizations. Learning about the relations between graphical representations, straightforward calculations and Maxwell's equations may equip students to blend their acquired understanding into an unambiguous story retelling the divergence and curl of electromagnetic fields.

\section{ACKNOWLEDGMENTS}

We would like to thank all the participating students whose cooperation made this research project possible. In addition, we gratefully acknowledge fruitful discussions with Diarmaid Hyland and Mossy Kelly at Dublin City University.
[1] L. Bollen, P. van Kampen, and M. De Cock, Students' difficulties with vector calculus in electrodynamics, Phys. Rev. ST Phys. Educ. Res. 11, 020129 (2015).

[2] C. Baily and C. Astolfi, Student Reasoning About the Divergence of a Vector Field, 2014 PERC Proceedings, Minneapolis, MN, July 30-31, 2014, edited by P. V. Engelhardt, A. D. Churukian, and D. L. Jones (AAPT, College Park, MD, 2015).

[3] C. Baily, L. Bollen, A. Pattie, P. van Kampen, and M. De Cock, Student thinking about the divergence and curl in mathematics and physics contexts, in 2015 PERC Proceedings, College Park, MD, July 29-30, 2015, edited by A. D. Churukian, D. L. Jones, and L. Ding (AAPT, College Park, MD, 2015), pp. 51-54.

[4] R. E. Pepper, S. V. Chasteen, S. J. Pollock, and K. K. Perkins, Observations on student difficulties with mathematics in upper-division electricity and magnetism, Phys. Rev. ST Phys. Educ. Res. 8, 010111 (2012).

[5] M. Artigue, J. Menigaux, and L. Viennot, Some aspects of students' conceptions and difficulties about differentials, Eur. J. Phys. 11, 262 (1990).

[6] F. R. Yeatts, Calculus and physics: Challenges at the interface, Am. J. Phys. 60, 716 (1992).

[7] E. F. Redish, Problem solving and the use of math in physics courses, in Presented in World View in Physics Education 2005: Focusing on Change, Delhi, 2005 (2005), http://www.compadre.org/per/items/detail.cfm?ID=3706.

[8] D. Nguyen and N. S. Rebello, Students' difficulties with integration in electricity, Phys. Rev. ST Phys. Educ. Res. 7, 010113 (2011).

[9] L. Doughty, E. McLoughlin, and P. van Kampen, What integration cues, and what cues integration in intermediate electromagnetism, Am. J. Phys. 82, 1093 (2014).
[10] B. R. Wilcox and S. J. Pollock, Upper-division student difficulties with the Dirac delta function, Phys. Rev. ST Phys. Educ. Res. 11, 010108 (2015).

[11] B. R. Wilcox and S. J. Pollock, Upper-division student difficulties with separation of variables, Phys. Rev. ST Phys. Educ. Res. 11, 020131 (2015).

[12] J. Larkin, J. McDermott, D. P. Simon, and H. A. Simon, Expert and novice performance in solving physics problems, Science 208, 1335 (1980).

[13] R. Karam, Framing the structural role of mathematics in physics lectures: A case study on electromagnetism, Phys. Rev. ST Phys. Educ. Res. 10, 010119 (2014).

[14] E. Kuo, M. M. Hull, A. Gupta, and A. Elby, How students blend conceptual and formal mathematical reasoning in solving physics problems, Sci. Educ. 97, 32 (2013).

[15] C. A. Manogue and T. Dray, Bridging the gap between mathematics and the physical sciences, APS Forum Educ., 13 (2004).

[16] M. D. Caballero, B. R. Wilcox, L. Doughty, and S.J. Pollock, Unpacking students' use of mathematics in upper-division physics: where do we go from here? Eur. J. Phys. 36, 065004 (2015).

[17] B. L. Sherin, How students understand physics equations, Cognit. Instr. 19, 479 (2001).

[18] D. Hu and N.S. Rebello, Using conceptual blending to describe how students use mathematical integrals in physics, Phys. Rev. ST Phys. Educ. Res. 9, 020118 (2013).

[19] J. Tuminaro, Ph.D. thesis, University of Maryland, College Park, MD, 2004.

[20] J. Tuminaro and E. F. Redish, Elements of a cognitive model of physics problem solving: Epistemic games, Phys. Rev. ST Phys. Educ. Res. 3, 020101 (2007). 
[21] D. C. Meredith and K. A. Marrongelle, How students use mathematical resources in an electrostatics context, Am. J. Phys. 76, 570 (2008).

[22] T. J. Bing and E. F. Redish, The cognitive blending of mathematics and physics knowledge, AIP Conf. Proc. 883, 26 (2007).

[23] T. J. Bing and E. F. Redish, Analyzing problem solving using math in physics: Epistemological framing via warrants, Phys. Rev. ST Phys. Educ. Res. 5, 020108 (2009).

[24] B. R. Wilcox, M. D. Caballero, D. A. Rehn, and S. J. Pollock, Analytic framework for students' use of mathematics in upper-division physics, Phys. Rev. ST Phys. Educ. Res. 9, 020119 (2013).

[25] E. F. Redish and E. Kuo, Language of Physics, Language of Math: Disciplinary Culture and Dynamic Epistemology, Sci. Educ. 24, 561 (2015).

[26] A. Van Heuvelen, Learning to think like a physicist: A review of research-based instructional strategies, Am. J. Phys. 59, 891 (1991).

[27] P. Kohl and N. Finkelstein, Student representational competence and self-assessment when solving physics problems, Phys. Rev. ST Phys. Educ. Res. 1, 010104 (2005).

[28] P. Kohl and N. Finkelstein, Effects of representation on students solving physics problems: A fine-grained characterization, Phys. Rev. ST Phys. Educ. Res. 2, 010106 (2006).

[29] P. Kohl and N. Finkelstein, Patterns of multiple representation use by experts and novices during physics problem solving, Phys. Rev. ST Phys. Educ. Res. 4, 010111 (2008).

[30] N. Khan, D. Hu, D. Nguyen, and N. S. Rebello, Assessing students' ability to solve introductory physics problems using integrals in symbolic and graphical representations, AIP Conf. Proc. 1413, 227 (2012).

[31] D. Nguyen and N. S. Rebello, Students' difficulties in transfer of problem solving across representations, AIP Conf. Proc. 1179, 221 (2009).

[32] M. De Cock, Representation use and strategy choice in physics problem solving, Phys. Rev. ST Phys. Educ. Res. 8, 020117 (2012).

[33] J. F. Wagner, C. A. Manogue, and J. R. Thompson, Representation issues: Using mathematics in upper-division physics, AIP Conf. Proc. 1413, 89 (2012).

[34] T. Fredlund, C. Linder, J. Airey, and A. Linder, Unpacking physics representations: Towards an appreciation of disciplinary affordance, Phys. Rev. ST Phys. Educ. Res. 10, 020129 (2014).

[35] G. Fauconnier and M. Turner, Conceptual integration networks, Cogn. Sci. 22, 133 (1998).

[36] G. Fauconnier and M. Turner, The Way We Think: "Conceptual Blending and the Mind's Hidden Complexities” (Basic Books, New York, 2002).
[37] G. Fauconnier and M. Turner, Conceptual blending, form and meaning, Recherches en communication 19, 57 (2003).

[38] D. Tall, The transition to formal thinking in mathematics, Math. Educ. Res. J. 20, 5 (2008).

[39] S. Coulson and T. Oakley, Blending basics, Cogn. Ling. 11, 175 (2001).

[40] N. S. Podolefsky and N. D. Finkelstein, Analogical scaffolding and the learning of abstract ideas in physics: Empirical studies, Phys. Rev. ST Phys. Educ. Res. 3, 020104 (2007).

[41] M. C. Wittmann, Using conceptual blending to describe emergent meaning in wave propagation, in Proceedings of the 9th Int. Conf. Learn. Sci., Vol. 1 (2010), pp. 659-666, arXiv:1008.0216.

[42] E. Gire and E. Price, Arrows as anchors: An analysis of the material features of electric field vector arrows, Phys. Rev. ST Phys. Educ. Res. 10, 020112 (2014).

[43] D. J. Griffiths, Introduction to Electrodynamics, 4th ed. (Addison-Wesley, Reading, MA, 2012).

[44] D. Tall and S. Vinner, Concept image and concept definition in mathematics with particular reference to limits and continuity, Educ. Stud. Math. 12, 151 (1981).

[45] A. Sfard, On the dual nature of mathematical conceptions: Reflections on processes and objects as different sides of the same coin, Educ. Stud. Math. 22, 1 (1991).

[46] C. Singh and A. Maries, Core graduate courses: A missed learning opportunity?, AIP Conf. Proc. 1513, 382 (2013).

[47] T. Dray and C. A. Manogue, The vector calculus gap: mathematics $\neq$ physics, PRIMUS 9, 21 (1999).

[48] M. Saarelainen, A. Laaksonen, and P. E. Hirvonen, Students' initial knowledge of electric and magnetic fields-more profound explanations and reasoning models for undesired conceptions, Eur. J. Phys. 28, 51 (2007).

[49] S. Törnkvist, K.-A. Pettersson, and G. Tranströmer, Confusion by representation: On student's comprehension of the electric field concept, Am. J. Phys. 61, 335 (1993).

[50] E. Gire and E. Price, Graphical representations of vector functions in upper-division E\&M, AIP Conf. Proc. 1413, 27 (2012).

[51] H. M. Schey, Div, Grad, Curl and All That: An Informal Text on Vector Calculus, 4th ed. (Norton, New York, 2004).

[52] D. Fleisch, A Student's Guide to Maxwell's Equations (Cambridge University Press, Cambridge, MA, 2008), p. 142 .

[53] R. P. Feynman, R. B. Leighton, and M. Sands, The Feynman Lectures on Physics, Vol. 2 (Addison-Wesley, Reading, MA, 1964), p. 536. 